\title{
A synthetic molecule stalls pre-mRNA splicing by enhancing cancer-relevant U2AF2 - RNA complexes
}

\author{
Clara L. Kielkopfa, Callen F. Feeney ${ }^{a, *}$, Rakesh Chatrikhi, ${ }^{a, *}$, Andrew MacRae ${ }^{b}$, \\ Georgios Alachouzos ${ }^{c}$, Zackary Falls ${ }^{d}$, Kholiswa M. Laird ${ }^{a}$, Jermaine L. Jenkins ${ }^{a}$, \\ Ram Samudralad ${ }^{d}$ Alison J. Frontier ${ }^{c}$, Melissa S. Jurica ${ }^{b}$ \\ aDepartment of Biochemistry and Biophysics, University of Rochester \\ School of Medicine and Dentistry, Rochester, NY, USA. \\ ${ }^{b}$ Department of Molecular and Cell Biology, University of California, Santa Cruz, CA, USA. \\ 'Department of Chemistry, University of Rochester, Rochester, NY, USA. \\ dDepartment of Bioinformatics, University at Buffalo, Buffalo, NY, USA. \\ *These authors contributed equally. \\ Email Contact: clara kielkopf@urmc.rochester.edu
}

Dysregulated pre-mRNA splicing is an emerging hallmark of cancers and hematologic malignancies, and renders these malignancies sensitive to spliceosome inhibition. As such, modulators of pre-mRNA splicing may offer as yet unrealized potential as anti-cancer therapeutics. Currently, the pre-mRNA splicing factor SF3b1 is the sole target of the major class of small molecule inhibitors of spliceosome assembly (e.g. sudemycin D6, E7107, H3B-8800). A heterodimer comprising U2AF2 and U2AF1 recognizes the $3^{\prime}$ splice site and initiates premRNA splicing. Whereas U2AF2 is an essential pre-mRNA splicing factor, U2AF1 plays an accessory role for splicing so-called "AG-dependent" sites. As such, a small molecule modulator of the U2AF2 - $3^{\prime}$ splice site complex may have general utility for treatment of cancers and hematologic malignancies.

Here, we report identification and characterization of a new small molecule that inhibits premRNA splicing by modulating U2AF2 - RNA complexes. We screened 1,593 structurally diverse compounds in a fluorescence polarization-based assay. For physiological relevance and statistical robustness of the polarization signal (which is proportional to molecular size), we prebound a U2AF2-U2AF1 ${ }^{\mathrm{S} 34 \mathrm{~F}}$-SF1 heterotrimer to a fluorescein (FI)-labeled splice site derived from the DEK oncogene. Although three hit compounds dissociated the ribonucleoprotein complex and inhibited pre-mRNA splicing in vitro, we considered these compounds lower priority due to nonspecific RNA binding. Remarkably, one hit compound specifically enhanced formation of the U2AF-containing ribonucleoprotein complex, yet inhibited in vitro splicing of representative substrates. Orthogonal BIAcore assays identified U2AF2 as the target subunit of this top hit compound. Structure-guided docking followed by mutagenesis established that the compound binds at a site between the tandem RNA recognition motifs of the splicing factor. Native gel electrophoresis further revealed that the compound stalled the pre-mRNA splicing process at the U2AF2-containing stage of spliceosome assembly.

Altogether, our results demonstrate a new means for molecular manipulation of pre-mRNA splicing: Locking spliceosome assembly at an early, inactive stage. Future optimized generations of this U2AF2-targeted compound could amplify the effects of SF3B1 modulators, reduce side effects, and/or increase efficacy in subsets of patients carrying U2AF1 or U2AF2 mutations or amplifications. 